\title{
New substitution bases for complexity classes
}

\author{
Stefano Mazzanti \\ Dipartimento di Culture del Progetto \\ Università Iuav di Venezia \\ Fondamenta delle Terese 2206, 30123 Venezia, Italy \\ email: mazzanti@iuav.it
}

\begin{abstract}
The set $A C^{0}(F)$, the $A C^{0}$ closure of $F$, is the closure with respect to substitution and concatenation recursion on notation of a set of basic functions comprehending the set $F$. By improving earlier work, we show that $A C^{0}(F)$ is the substitution closure of a simple function set and characterize well-known function complexity classes as the substitution closure of finite sets of simple functions.
\end{abstract}

\section{Introduction}

A finite function set $F$ is a substitution basis for a function class $G$ (and $G$ is the substitution closure of $F$ ) when $G$ can be defined using only the functions in $F$, the projection functions and the substitution operator. Several function classes like partial recursive functions, Grzegorczyk classes $\mathcal{E}_{n}$ for $n \geq 2$ and polynomial time computable functions have a substitution basis, see [6] for a list of references. But such bases may contain awkward functions.

A nice example of basis for a non-trivial function class was given in $[7,8]$ where the set $\left\{x+y, x \dot{-} y, x \wedge y,\lfloor x / y\rfloor, 2^{|x|^{2}}\right\}$ was shown to be a basis for the class $T C^{0}$ of functions computable by polysize, constant depth threshold circuits. ${ }^{1}$

Subsequently, the existence of plain bases was considered for the set $A C^{0}(F)$, the closure with respect to substitution and concatenation recursion on notation $(\mathrm{CRN})$ of a set of basic functions comprehending the set $F .{ }^{2}$ In [5], it was shown that $A C^{0}(F)$ admits a basis, provided that it contains integer division. From this result, the above mentioned basis for $T C^{0}$ was obtained. Later, the existence of a basis for $A C^{0}(F)$ was stated without assuming any hypothesis and a basis for $A C^{0}$ was introduced [6].

However, the basis for $T C^{0}$ depends on the fact that integer division is in $T C^{0}$, which is a hard result to show [4], and the basis for $A C^{0}$ contains some

\footnotetext{
${ }^{1} x \wedge y$ is the bitwise and of $x$ and $y$. Names $A C^{0}, T C^{0}, N C^{1}$ are usually intended to denote language classes. However, in this paper they will always denote function classes, since no misunderstanding is possible.

${ }^{2} A C^{0}(F)$ is an obvious extension of Clote's characterization of $A C^{0}$ functions ([2]) obtained by adding the functions in $F$ to the set of basic functions. For example, in [3] the set of $T C^{0}$ functions has been defined as $A C^{0}$ (mult) where mult is the multiplication operation.
} 
non-standard arithmetical functions which handle their arguments as sequence encodings.

This paper tries to eliminate these drawbacks and improves the results of [5] and [6]. New bases for $A C^{0}, T C^{0}$ and other complexity classes are obtained in a new, uniform and division-independent way by exploiting elementary properties of geometric series.

In the Preliminaries, the basic definitions and the main results of [5] and [6] are recalled.

Section 3 introduces a basis for $A C^{0}(F)$ that depends on a function parameter.

Then, by setting such function in two different ways, in Section 4 we obtain a new basis for $A C^{0}(F)$ which yields immediately a new basis for $A C^{0}$, and in Section 5 we obtain two new bases for $T C^{0}$.

Finally, following [6], we derive new bases for $N C^{1}, L, P$ and $P S P A C E$ computable functions.

Even if the results of this paper may seem just aesthetic improvements, they shed some light on the difference between $A C^{0}$ and $T C^{0}$ and could possibly lead to a new, algebraic proof that $A C^{0} \neq T C^{0}$. Indeed, both $A C^{0}$ and $T C^{0}$ have a basis of six functions which differ for one function only.

\section{Preliminaries}

In this paper, we will only consider functions with finite arity on the set $\mathbb{N}=\{0,1, \ldots\}$ of natural numbers.

From now on, we agree that $x, y, z, u, v, w, i, j, k, l, n, m, r$ range over $\mathbb{N}$, that $a, b, c$ range over positive integers, that $\mathbf{x}, \mathbf{y}$ range over sequences (of fixed length) of natural numbers, that $p, q$ range over integer polynomials with non negative values and that $f, g, h$ range over functions.

A function $f$ is a polynomial growth function iff there is a polynomial $p$ majorizing (the length of) $f$, i.e. such that $|f(\mathbf{x})| \leq p(|\mathbf{x}|)$ or, equivalently, $f(\mathbf{x})<2^{p(|\mathbf{x}|)}$ for any $\mathbf{x}$, where $\left|x_{1}, \ldots, x_{n}\right|=\left|x_{1}\right|, \ldots,\left|x_{n}\right|$ and $|x|=\left\lceil\log _{2}(x+\right.$ $1)\rceil$ is the number of bits of the binary representation of $x$.

We will use the following unary functions: the binary successor functions $s_{0}$ : $x \longmapsto 2 x$ and $s_{1}: x \longmapsto 2 x+1$; the constant functions $C_{y}: x \longmapsto y$; the signum function $s g: x \longmapsto \min (x, 1)$; the cosignum function $\cos g: x \longmapsto 1-s g(x)$; the quadratum function quad $: x \longmapsto x^{2}$; length function len $: x \longmapsto|x|$; the unary smash function us : $x \longmapsto 2^{|x|^{2}}$; the next power of two function pow : $x \longmapsto 2^{|x|}$.

We will also use the following functions: the addition function add :x,y $x+y$; the multiplication function mult $: x, y \longmapsto x y$; the modified subtraction function $s u b: x, y \longmapsto x-y=\max (x-y, 0)$; the division function quot $: x, y \longmapsto$ $\lfloor x / y\rfloor$; the remainder function rem : $x, y \longmapsto x-y\lfloor x / y\rfloor$; the conditional function

$$
\operatorname{cond}(x, y, z)=\left\{\begin{array}{ll}
y & \text { if } x=0 \\
z & \text { otherwise }
\end{array} ;\right.
$$

the bit function bit : $x, y \longmapsto r e m\left(\left\lfloor x / 2^{y}\right\rfloor, 2\right)$; the multiplication by a power function multp : $x, y \longmapsto x 2^{|y|}$; the concatenation function conc $: x, y \longmapsto$ $x * y=x 2^{|y|}+y$; the smash function smash : $x, y \longmapsto x \# y=2^{|x| \cdot|y|}$ ; the most significant part function $M S P: x, y \longmapsto\left\lfloor x / 2^{y}\right\rfloor$; the log most 
significant part function $\left.m s p: x, y \longmapsto \mid x / 2^{|y|}\right\rfloor$; the least significant part function $L S P: x, y \longmapsto \operatorname{rem}\left(x, 2^{y}\right)$; the log least significant part function $l s p: x, y \longmapsto \operatorname{rem}\left(x, 2^{|y|}\right)$. A fundamental role will be played by the bitwise and function and $: x, y \longmapsto x \wedge y$ such that $\operatorname{bit}(x \wedge y, i)=\operatorname{bit}(x, i) \cdot \operatorname{bit}(y, i)$ for any $i$.

For $l, n>0$, let $\left\langle x_{n}, \ldots, x_{1} ; l\right\rangle=\sum_{i<n} x_{i+1} 2^{l i}$; if $x_{n}, \ldots, x_{1}<2^{l}$ then $x_{n}, \ldots, x_{1}$ are the base $2^{l}$ digits of $\left\langle x_{n}, \ldots, x_{1} ; l\right\rangle$. Then, we will also use the functions arl, ar $2 l$, repl, convl such that

$$
\begin{aligned}
\operatorname{arl}(l) & =\sum_{i<|l|} i 2^{|l| i}, \\
\operatorname{ar} 2 l(l) & =\sum_{i<|l|} i^{2} 2^{|l| i} \\
\operatorname{repl}(x, l, n) & =\sum_{i<|n|} \operatorname{rem}\left(x, 2^{|l|}\right) 2^{|l| i} \\
\operatorname{convl}(x, l, r, n) & =\sum_{i<|n|} \operatorname{rem}\left(x_{i+1}, 2^{|r|}\right) 2^{|r| i}
\end{aligned}
$$

where $x_{|n|}, \ldots, x_{1}$ are the $|n|$ least significant base $2^{|l|}$ digits of $x$. All the functions above return 0 when one of $l, r, n$ is 0 .

Note that

$$
\operatorname{repl}(x, l, n)=\langle\overbrace{x, \ldots, x}^{|n|-\text { times }} ;|l|\rangle
$$

for $x<2^{|l|}$ and

$$
\operatorname{convl}(\langle\mathbf{x} ;|l|\rangle, l, r, n)=\langle\mathbf{x} ;|r|\rangle
$$

where $\mathbf{x}=x_{|n|}, \ldots, x_{1}$ with $x_{i}<2^{\min (|l|,|r|)}$ for $1 \leq i \leq n$.

As usual, the characteristic function of a predicate $Q$ on natural numbers is the function $f(\mathbf{x})$ returning 1 if $Q(\mathbf{x})$ is true, 0 otherwise. We say that a predicate is in a class $F$ of functions, meaning that its characteristic function is in $F$.

Let

$$
r p(x, l, n)= \begin{cases}x \cdot \sum_{i<|n|} 2^{|l| i} & \text { if } A C^{0} \_S U M(x, l, n) \\ 0 & \text { otherwise }\end{cases}
$$

where

$$
A C^{0}{ }_{-} S U M(x, l, n) \Leftrightarrow(\ln >0) \wedge\left(\bigvee_{i=1}^{3} P_{i}(x, l, n)\right)
$$

and $P_{1}, P_{2}$, and $P_{3}$ are respectively the following predicates

$$
\begin{aligned}
& P_{1}(x, l, n) \Leftrightarrow x=\langle\overbrace{1, \ldots, 1}^{|l|-\text { times }} ;|l|\rangle \wedge 1<l, \\
& P_{2}(x, l, n) \Leftrightarrow x=\langle|l|-1, \ldots, 1,0 ;|l|\rangle \wedge 1<l, \\
& P_{3}(x, l, n) \Leftrightarrow x<2^{|l||n|} \wedge \forall_{i<j<|n|}\left(x 2^{|l| i} \wedge x 2^{|l| j}=0\right) .
\end{aligned}
$$


As we will see in Section 4, the predicate $A C^{0} \quad S U M$ guarantees that the function $r p$ is in $A C^{0}$, even if $\left.x \cdot \sum_{i<|n|}\right|^{|l| i}$ is in $\bar{T} C^{0}-A C^{0}$.

Finally, we will use the following operators on functions:

- the substitution operator $\operatorname{SUBST}\left(g_{1}, \ldots, g_{b}, h\right)$ transforming functions $g_{1}, \ldots, g_{b}: \mathbb{N}^{a} \rightarrow \mathbb{N}$ and function $h: \mathbb{N}^{b} \rightarrow \mathbb{N}$ into the function $f: \mathbb{N}^{a} \rightarrow \mathbb{N}$ such that $f(\mathbf{x})=h\left(g_{1} \mathbf{x}, \ldots, g_{b} \mathbf{x}\right)$;

- the concatenation recursion on notation operator $C R N\left(g, h_{0}, h_{1}\right)$ transforming functions $h_{0}: \mathbb{N}^{a+1} \rightarrow \mathbb{N}$ and $h_{1}: \mathbb{N}^{a+1} \rightarrow \mathbb{N}$ with values in $\{0,1\}$ and function $g: \mathbb{N}^{a} \rightarrow \mathbb{N}$ into the function $f: \mathbb{N}^{a+1} \rightarrow \mathbb{N}$ such that

$$
\begin{aligned}
f(0, \mathbf{y}) & =g(\mathbf{y}), \\
f\left(s_{i}(x), \mathbf{y}\right) & =s_{h_{i}(x, \mathbf{y})}(f(x, \mathbf{y}))
\end{aligned}
$$

where in the second equation $i \in\{0,1\}$ and $x>0$ when $i=0$.

For any set $F$ of functions, let $\operatorname{clos}_{S U B S T}(F)$ be the closure under substitution of $F \cup I$ where $I$ is the set of the projection functions

$$
I^{a}[i]: \quad x_{1}, \ldots, x_{a} \longmapsto x_{i} \quad(1 \leq i \leq a)
$$

with any arity $a$. In the following, we will abuse the notation above as usual and we will write $\operatorname{clos}_{S U B S T}\left(f_{1}, \ldots, f_{n}, G\right)$ when $F=\left\{f_{1}, \ldots, f_{n}\right\} \cup G$ (the sequence $f_{1}, \ldots, f_{n}$ may be empty and the set $G$ may be omitted).

For any set $F$ of polynomial growth functions, we define $A C^{0}(F)$, the $A C^{0}$ closure of $F$, as the closure under substitution and CRN of $\left\{C_{0}, s_{0}, s_{1}\right.$, smash, len, bit $\} \cup F \cup I^{3}$.

The class $A C^{0}$ of functions computable by polysize, constant depth, unbounded fan-in Boolean circuits, the class $T C^{0}$ of functions computable by polysize, constant depth, unbounded fan-in threshold circuits, the class $N C^{1}$ of functions computable by polysize, logarithmic depth, bounded fan-in Boolean circuits have been characterized using substitution and CRN $[1,2,3]$ :

$$
A C^{0}=A C^{0}(\emptyset), T C^{0}=A C^{0}(\text { mult }), N C^{1}=A C^{0}(\text { tree })
$$

where tree is a unary function taking values in $\{0,1\}$ such that tree $(x)$ is the value of the and/or tree with or gate at the root represented by $x$ when $|x|=$ $4^{n}+1>1$. E.g. for $x=10110_{2}$ we have tree $(x)=0=(0 \wedge 1) \vee(1 \wedge 0)$. For a definition of tree see [1] or the Appendix of [5].

If $F$ is a class of functions such that $F=\operatorname{clos}_{S U B S T}\left(f_{1}, \ldots, f_{a}\right)$ then $\left\{f_{1}, \ldots, f_{a}\right\}$ is a (substitution or superposition) basis for $F$ and $F$ is the substitution closure of $\left\{f_{1}, \ldots, f_{a}\right\}$.

For any function $f: \mathbb{N}^{a} \longrightarrow \mathbb{N}$, we define the function $f^{\dagger}$, the canonical dagger of $f$, by setting $f^{\dagger_{c}}\left(x_{1}, \ldots, x_{a}, l, n\right)=0$ if $l=0$ or $n=0$ or $x_{i} \geq 2^{|l||n|}$ for some $1 \leq i \leq a$ and

$$
\begin{aligned}
f^{\dagger_{c}}\left(x_{1}, \ldots, x_{a}, l, n\right) & \\
& =\left\langle\operatorname{rem}\left(f\left(x_{1,|n|}, \ldots, x_{a,|n|}\right), 2^{|l|}\right), \ldots, \operatorname{rem}\left(f\left(x_{1,1}, \ldots, x_{a, 1}\right), 2^{|l|}\right) ;|l|\right\rangle
\end{aligned}
$$

\footnotetext{
${ }^{3}$ See [6] for a discussion about the relationship of our definition of $A C^{0}(F)$ and similar definitions given in the literature.
} 
when $l, n>0, x_{1}=\left\langle x_{1,|n|}, \ldots, x_{1,1} ;|l|\right\rangle, \ldots, x_{a}=\left\langle x_{a,|n|}, \ldots, x_{a, 1} ;|l|\right\rangle$ and $x_{i, j}<2^{|l|}$ for $1 \leq i \leq a$ and $1 \leq j \leq|n|$. Note that the equation above reduces to

$$
f^{\dagger^{\prime}}\left(x_{1}, \ldots, x_{a}, l, n\right)=\left\langle f\left(x_{1,|n|}, \ldots, x_{a,|n|}\right), \ldots, f\left(x_{1,1}, \ldots, x_{a, 1}\right) ;|l|\right\rangle
$$

if $f\left(x_{1, j}, \ldots, x_{a, j}\right)<2^{|l|}$ for $1 \leq j \leq|n|{ }^{4}$

In [5], the following result has been obtained.

Quotient Basis Theorem. For any set $F$ of polynomial growth functions, if quot $\in A C^{0}(F)$ then

$$
A C^{0}(F)=\cos _{S U B S T}\left(a d d, \text { sub, and, quot, us, } F^{\dagger c}\right)
$$

where $F^{\dagger_{c}}=\left\{f^{\dagger^{\dagger}} \mid f \in F\right\}$.

The theorem above enabled us to show that $\{a d d, s u b$, and,quot,us $\}$ is a basis for $T C^{0}$ by noting that quad ${ }^{\dagger c} \in \operatorname{clos}_{S U B S T}(a d d, s u b$, and, quot, us) and to show that $\left\{a d d, s u b\right.$, and, quot, us, tree $\left.^{\dagger_{c}}\right\}$ is a basis for $N C^{1}$.

Later, in [6], we improved the method of CRN elimination introduced in [5] and proved the following Quotient-free Basis Theorem which states that, for any finite set $F$ of polynomial growth functions, the set $A C^{0}(F)$ has a basis. From the Quotient-free Basis Theorem we obtained a new basis for $A C^{0}$ by setting $F=\emptyset$.

Quotient-Free Basis Theorem. For any set $F$ of polynomial growth functions,

$$
A C^{0}(F)=\operatorname{clos}_{S U B S T}\left(C_{1}, \text { add, sub, and, conc, len, msp, ar } 2 l, \text { repl, convl, } F^{\dagger c}\right) .
$$

In this paper we will show the following improved version of the Quotient-free Basis Theorem.

Improved Quotient-free Basis Theorem. For any set $F$ of polynomial growth functions,

$$
A C^{0}(F)=\cos _{S U B S T}\left(C_{1}, a d d, s u b, \text { and, } m s p, r p, F^{\dagger c}\right) .
$$

The Improved Quotient-free Basis Theorem yields immediately a new basis for $A C^{0}$.

Corollary 1. $A C^{0}=\operatorname{clos}_{S U B S T}\left(C_{1}, a d d\right.$, sub, and, $\left.m s p, r p\right)$.

Moreover, we will state the following characterizations of $T C^{0}$.

\section{Theorem 2.}

$$
\begin{aligned}
T C^{0} & =\operatorname{clos}_{S U B S T}\left(C_{1}, \text { add, sub, and, msp, } x \cdot \sum_{i<|n|} 2^{|l| i}\right) \\
& =\operatorname{clos}_{\text {SUBST }}\left(C_{1}, \text { add, sub, and, msp, } \sum_{i<|n|} 2^{|l| i}, \text { quad }\right) .
\end{aligned}
$$

\footnotetext{
${ }^{4}$ See [6] for a full account about dagger operators.
} 


\section{A parametric Quotient-free Basis Theorem for $A C^{0}(F)$}

Let $r p t$ be any function such that

$$
\operatorname{rpt}(x, l, n)=\operatorname{rp}(x, l, n)=x \cdot \sum_{i<|n|} 2^{|l| i}
$$

when $A C^{0}$ _SUM $(x, l, n)$ is true. In this section, for any set $F$ of polynomial growth functions, we will show that

$$
A C^{0}(F) \subseteq \operatorname{clos}_{S U B S T}\left(C_{1}, \text { add, sub, and, msp, rpt, } F^{\dagger c}\right)
$$

and if $r p t \in A C^{0}(F)$ then

$$
A C^{0}(F)=\operatorname{clos}_{S U B S T}\left(C_{1}, \text { add, sub, and, msp, rpt, } F^{\dagger c}\right) .
$$

The basic idea of the proof is that the functions repl,arl,ar2l and convl are special instances of $x \cdot \sum_{i<|n|} 2^{|l| i}$ and can be obtained from $\operatorname{rpt}(x, l, n)$ by substituting a suitable $A C^{0}$ function for $x$.

A normal function class is a function class closed with respect to substitution which contains the function set $I \cup\left\{C_{1}, a d d, s u b, a n d, m s p, r p t\right\}$. Moreover, a function is normal iff it belongs to every normal class or, equivalently, iff it belongs to $\operatorname{clos}_{S U B S T}\left(C_{1}\right.$, add, sub, and, msp, rpt).

In the following, we will show that $r e p l$, arl, ar $2 l$ and convl are normal functions and so by the Quotient-free Basis Theorem, any normal function class contains $A C^{0}(F)$ if it contains $F^{\dagger c}$.

Lemma 3. If $x<2^{|l|}$ then $\forall_{i<j<|n|}\left(x 2^{|l| i} \wedge x 2^{|l| j}=0\right)$ and

$$
\operatorname{rpt}(x, l, n)=\langle\overbrace{x, \ldots, x}^{|n| \text {-times }} ;|l|\rangle .
$$

Lemma 4. The following functions are normal: $C_{n}$ for any $n$, $s g, \operatorname{cosg}, s_{0}, s_{1}$, pow, smash, $2^{|x|-|y|}$, multp, conc, lsp, and for any polynomial $p$, $2^{p(|\mathbf{x}|)}$.

Proof. The proof is similar to that of Lemma 3 in [6]. Note first that

$$
C_{0}(x)=C_{1}(x) \dot{-} C_{1}(x), C_{n+1}(x)=C_{n}(x)+C_{1}(x)
$$

and

$$
\operatorname{cosg}(x)=C_{1}(x) \stackrel{\bullet}{-} x, s g(x)=\operatorname{cosg}(\cos g(x))
$$


Then,

$$
\begin{aligned}
s_{0}(x) & =x+x, s_{1}(x)=s_{0}(x)+C_{1}(x), \\
\operatorname{pow}(x) & =2^{|x|}=\operatorname{cosg}(x)+(\operatorname{rpt}(1, x, 2) \dot{-} 1), \\
\operatorname{smash}(x, y) & =2^{|x||y|}=\operatorname{rpt}\left(2^{|x|} \dot{-} 1, x, y\right)+1, \\
\max (x, y) & =(x \dot{-} y)+y, \\
x 2^{|\max (x, y)|} & =\operatorname{rpt}(x, \max (x, y), 2) \dot{-} x, \\
2^{|x|}|\dot{-}| y \mid & =\left\lfloor 2^{|x|} / 2^{|y|}\right\rfloor+\operatorname{sg}(|y| \dot{-}|x|)=m s p\left(2^{|x|}, 2^{|y|}-1\right)+s g\left(2^{|x|} \dot{-} 2^{|y|}\right) \\
\operatorname{multp}(x, y) & =\left\lfloor x 2^{|\max (x, y)|} / 2^{|x|-|y|}\right\rfloor=m s p\left(x 2^{|\max (x, y)|}, 2^{|x| \dot{-}|y|}-1\right), \\
\operatorname{conc}(x, y) & =\operatorname{multp}(x, y)+y, \\
\operatorname{lsp}(x, y) & =x \dot{-} \operatorname{multp}(\operatorname{msp}(x, y), y) .
\end{aligned}
$$

The function $2^{p(|\mathbf{x}|)}$ is normal for all polynomials $p$ with non negative coefficients, because the function $2^{p(|\mathbf{x}|)}$ can be obtained from the normal functions $2^{c}$ and $2^{|x|}$ by a finite number of applications of the two equations $2^{p(|\mathbf{x}|) q(|\mathbf{y}|)}=\left(2^{p(|\mathbf{x}|)}-\right.$ 1)\#( $\left(2^{q(|\mathbf{y}|)}-1\right)$ and $2^{p(|\mathbf{x}|)+q(|\mathbf{y}|)}=2^{\left|\left(2^{p(|\mathbf{x}|)}-1\right) *\left(2^{q(|\mathbf{y}|)}-1\right)\right|}$. Moreover, $2^{p(|\mathbf{x}|)}$ is normal even for any integer polynomial $p$ with non negative values. Indeed, $p$ can be expressed as the modified subtraction of two polynomials $q, q^{\prime}$ with non negative coefficients such that $q(|\mathbf{x}|) \geq q^{\prime}(|\mathbf{x}|)$ and we obtain that $2^{p(|\mathbf{x}|)}=$ $2^{q(|\mathbf{x}|) \dot{-} q^{\prime}(|\mathbf{x}|)}=m s p\left(2^{q(|\mathbf{x}|)}, 2^{q^{\prime}(|\mathbf{x}|)}-1\right)+s g\left(2^{q(|\mathbf{x}|)} \dot{-} 2^{q^{\prime}(|\mathbf{x}|)}\right)$.

Lemma 5. Function cond is normal.

Proof. By Lemma 4 the function $2^{|y|+|z|}$ is normal. Then, also the function

$$
f(x, y, z)=\operatorname{rpt}\left(\operatorname{sg}(x), 1,2^{|y|+|z|}-1\right)= \begin{cases}0 & \text { if } x=0, \\ 2^{|y|+|z|}-1 & \text { otherwise }\end{cases}
$$

is normal. The lemma follows immediately by noting that

$$
\operatorname{cond}(x, y, z)=\operatorname{and}(f(x, y, z), z)+\operatorname{and}(f(\cos g(x), y, z), y) .
$$

Any normal class is closed with respect to definition by cases and contains the predicates generated by the standard comparison predicates and the Boolean connectives.

Lemma 6. Any normal class is closed with respect to definition by cases.

Proof. Assume that $C$ is a normal class and $f_{1}, \ldots, f_{a+1} \in C$. Let $g_{1}, \ldots, g_{a} \in C$ be the characteristic functions of $Q_{1}, \ldots, Q_{a}$, respectively. The lemma follows immediately from Lemma 5 because the function

$$
f(\mathbf{x})= \begin{cases}f_{1}(\mathbf{x}) & \text { if } Q_{1}(\mathbf{x}), \\ \ldots & \ldots \\ f_{a}(\mathbf{x}) & \text { if } Q_{a}(\mathbf{x}) \\ f_{a+1}(\mathbf{x}) & \text { otherwise }\end{cases}
$$


can be defined as

$$
f(\mathbf{x})=\operatorname{cond}\left(g_{1}(\mathbf{x}), \operatorname{cond}\left(\ldots \operatorname{cond}\left(g_{a}(\mathbf{x}), f_{a+1}(\mathbf{x}), f_{a}(\mathbf{x})\right) \ldots\right), f_{1}(\mathbf{x})\right) .
$$

Lemma 7. The predicates of a normal class are closed with respect to conjunction, disjunction, and negation.

Proof. Assume that $C$ is a normal class and let $g_{1} \in C$ and $g_{2} \in$ $C$ be the characteristic functions of predicates $Q_{1}$ and $Q_{2}$, respectively. Then, $\cos g\left(g_{1}(x)\right), \operatorname{cond}\left(g_{1}(x), C_{0}(x), \operatorname{cond}\left(g_{2}(x), C_{0}(x), C_{1}(x)\right)\right)$ and cond $\left(g_{1}(x), \operatorname{cond}\left(g_{2}(x), C_{0}(x), C_{1}(x)\right), C_{1}(x)\right)$ are the characteristic functions of $\neg Q_{1}, Q_{1} \wedge Q_{2}$ and $Q_{1} \vee Q_{2}$, respectively. The lemma follows immediately from Lemma 4 and Lemma 5 .

Lemma 8. The comparison predicates $x<y, x \leq y, x>y, x \geq y, x=y, x \neq y$ are normal.

Proof. Note that $x>y \Leftrightarrow s g(x \dot{-} y)=1$ and $x=y \Leftrightarrow \cos g((x \dot{-} y)+(y \dot{-} x))=1$. The remaing predicates can be defined using the Boolean operations and the lemma follows from Lemma 7 .

Lemma 9. Function repl is normal.

Proof. Since $l s p(x, l)=\operatorname{rem}\left(x, 2^{|l|}\right)<2^{|l|}$, by Lemma 3

$$
\begin{aligned}
\operatorname{repl}(x, l, n) & =\langle\overbrace{\operatorname{rem}\left(x, 2^{|l|}\right), \ldots, \operatorname{rem}\left(x, 2^{|l|}\right)}^{|n| \text {-times }} ;|l|\rangle \\
& = \begin{cases}\operatorname{rpt}(l \operatorname{sp}(x, l), l, n) & \text { if }(l>0) \wedge(n>0), \\
0 & \text { otherwise }\end{cases}
\end{aligned}
$$

The lemma follows immediately by Lemmata $6-8$.

Lemma 10. If $\sum_{j=1}^{N} x_{j}<2^{L}$ then

$$
\left\langle\sum_{j=N-1}^{N} x_{j}, \ldots, \sum_{j=2}^{N} x_{j}, \sum_{j=1}^{N} x_{j}, \ldots, \sum_{j=1}^{1} x_{j} ; L\right\rangle=\left\langle x_{N}, \ldots, x_{1} ; L\right\rangle \cdot \sum_{i<N} 2^{L i} .
$$

Lemma 11. Functions arl and $|x|$ are normal.

Proof. Set $L=|l|$ and consider the normal function $f(l)=\operatorname{rpt}(\operatorname{rpt}(1, l, l), l, l)$. By definition of rpt and Lemma 10

$$
f(l)=\langle\overbrace{1, \ldots, 1}^{L-\text { times }} ; L\rangle \cdot \sum_{i<L} 2^{L i}=\langle 1, \ldots, L \dot{-} 1, L, L \dot{-} 1, \ldots, 1 ; L\rangle .
$$

Therefore, $\operatorname{arl}(l)=l s p\left(f(l), 2^{|l|^{2}}-1\right) \dot{-} r p t(1, l, l)$ because $l s p\left(f(l), 2^{|l|^{2}}-1\right)=$ $\langle L, \ldots, 1 ; L\rangle$ and $\operatorname{arl}$ is normal because $l \operatorname{sp}\left(x, 2^{|l|^{2}}-1\right)$ is normal by Lemma 4 . Finally, $|x|=\operatorname{lsp}\left(\operatorname{msp}\left(f(x), 2^{|x|^{2}-|x|}-1\right), x\right)$ and $|x|$ is normal because $l s p, 2^{|x|^{2}-|x|}$ and $2^{|x|}$ are normal by Lemma 4 . 
Lemma 12. Function ar $2 l$ is normal.

Proof. Set $L=|l|$ and consider the function $f(l)=\operatorname{rpt}(\operatorname{arl}(l), l, l)$. By definition of $r p t$ and Lemma 10

$$
\begin{aligned}
f(l)=\langle L \dot{-} 1, \ldots, 1,0 ; L\rangle & \cdot \sum_{i<L} 2^{L i} \\
& =\left\langle t_{L \dot{-1}} \dot{-} t_{L-2}, \ldots, t_{L \dot{-1}} \dot{-} t_{2}, t_{L-1} \dot{-}_{1}, t_{L \dot{-1}}, \ldots, t_{1}, t_{0} ; L\right\rangle
\end{aligned}
$$

where $t_{n}=\frac{n(n+1)}{2}$ is the $n$-th triangular number.

Now, since $2 t_{n}-n=n^{2}$, we obtain $\operatorname{ar} 2 l(l)=2 \cdot l \operatorname{sp}\left(f(l), 2^{|l|^{2}}-1\right) \dot{-} \operatorname{arl}(l)$ because $l s p\left(f(l), 2^{|l|^{2}}-1\right)=\left\langle t_{L-1}, \ldots, t_{1}, t_{0} ; L\right\rangle$ and $\operatorname{ar} 2 l$ is normal because $\operatorname{arl}$ and $l s p\left(x, 2^{|l|^{2}}-1\right)$ are normal by the lemma above and Lemma 4 .

The function $\operatorname{incr}(x, l, r, n)=\operatorname{rpt}\left(x, 2^{|r| \dot{-}|l|}, n\right) \wedge \operatorname{rpt}\left(2^{|l|}-1, r, n\right)$ has been introduced in [8]. The following lemma is analogous to Lemma 2.10 of [5] and Statement 1.1.4.3 of [8].

Lemma 13. If $l, n>0,|r| \geq(|n|+1)|l|$ and $x_{|n|}, \ldots, x_{1}<2^{|l|}$ then

$$
\operatorname{incr}\left(\left\langle x_{|n|}, \ldots, x_{1} ;|l|\right\rangle, l, r, n\right)=\left\langle x_{|n|}, \ldots, x_{1} ;|r|\right\rangle .
$$

Lemma 14. Function incr is normal.

Proof. The lemma follows immediately from Lemma 4.

Lemma 15. Function convl is normal.

Proof. Set $L=|l|, R=|r|$ and $N=|n|$. We first define a function decr such that $\operatorname{decr}\left(\left\langle x_{N}, \ldots, x_{1} ; L\right\rangle, l, r, n\right)=\left\langle x_{N}, \ldots, x_{1} ; R\right\rangle$ provided that $R<L$ and $x_{N}, \ldots, x_{1}<2^{R}$.

For $x=\left\langle x_{N}, \ldots, x_{1} ; L\right\rangle$, we have

$$
\begin{aligned}
\operatorname{incr}\left(x, l, 2^{L(N+1)+R}-1, n\right) & =\left\langle x_{N}, \ldots, x_{2}, x_{1} ; L(N+1)+R\right\rangle \\
& =\left\langle x_{N} 2^{R(N-1)}, \ldots, x_{2} 2^{R}, x_{1} ; L(N+1)\right\rangle
\end{aligned}
$$

by Lemma 13 and Lemma 20 of [6].

Now, for $y=\left\langle x_{N} 2^{R(N-1)}, \ldots, x_{2} 2^{R}, x_{1} ; L(N+1)\right\rangle$, we have

$$
i<j<N \Rightarrow y 2^{L(N+1) i} \wedge y 2^{L(N+1) j}=0
$$

because

$$
\operatorname{bit}\left(y 2^{L(N+1) i}, k\right)=\left\{\begin{array}{cl}
0 & \text { if } k<L(N+1) i \\
\operatorname{bit}\left(x_{q} 2^{R q}, s\right) & \text { otherwise }
\end{array}\right.
$$

where

$$
q=\lfloor k-L(N+1) i) / L(N+1)\rfloor=\lfloor k / L(N+1)\rfloor-i
$$

and

$$
s=\operatorname{rem}(k-L(N+1) i), L(N+1))=\operatorname{rem}(k, L(N+1)) .
$$


So, if

$$
b i t\left(y 2^{L(N+1) i}, k\right)=b i t\left(y 2^{L(N+1) j}, k\right)=1
$$

then

$$
\operatorname{bit}\left(x_{q} 2^{R q}, s\right)=\operatorname{bit}\left(x_{p} 2^{R p}, s\right)=1
$$

with $p<q$. But this means that $R p \leq s<R p+R$ and $R q \leq s<R q+R$ which implies $s<R(p+1) \leq R q \leq s$, a contradiction.

Therefore, by Lemma 10, the function

$$
f(x, l, r, n)=\operatorname{rpt}\left(\operatorname{incr}\left(x, l, 2^{L(N+1)+R}-1, n\right), 2^{L(N+1)}-1, n\right)
$$

satisfies the following equations

$$
\begin{aligned}
f(\langle\mathbf{x} ; L\rangle, l, r, n) & =\left\langle x_{N} 2^{R(N-1)}, \ldots, x_{2} 2^{R}, x_{1} ; L(N+1)\right\rangle \cdot \sum_{i<N} n 2^{L(N+1) i} \\
= & \left\langle\langle x_{N} \overbrace{0, \ldots, 0}^{N-1} ; R\rangle, \ldots,\left\langle x_{N}, \ldots, x_{2}, 0 ; R\right\rangle,\right. \\
& \left.\left\langle x_{N}, \ldots, x_{1} ; R\right\rangle, \ldots,\langle\overbrace{0, \ldots, 0}^{N-1}, x_{1} ; R\rangle ; L(N+1)\right\rangle
\end{aligned}
$$

where $\mathbf{x}=x_{N}, \ldots, x_{1}$. Then, for

$$
\operatorname{decr}(x, l, r, n)=l \operatorname{sp}\left(m s p\left(f(x, l, n), 2^{L(N+1)(N-1)}-1\right), 2^{R N}-1\right)
$$

we have $\operatorname{decr}\left(\left\langle x_{N}, \ldots, x_{1} ; L\right\rangle, l, r, n\right)=\left\langle x_{N}, \ldots, x_{1} ; R\right\rangle$. Moreover, decr is normal because $2^{L(N+1)(N-1)}-1$ and $2^{R N}-1$ are normal by Lemma 4 and $f$ is normal by Lemma 14 and Lemma 4 .

Furthermore, define the function

$$
\operatorname{trim}(x, l, r, n)= \begin{cases}x \wedge \operatorname{rpt}\left(2^{R}-1, l, n\right) & \text { if } L \geq R>0 \\ 0 & \text { otherwise }\end{cases}
$$

and note that for $R \leq L$ we have

$$
\operatorname{trim}(x, l, r, n)=\left\langle\operatorname{rem}\left(x_{N}, 2^{R}\right), \ldots, \operatorname{rem}\left(x_{1}, 2^{R}\right) ; L\right\rangle
$$

where $x_{N}, \ldots, x_{1}$ are the $N$ least significant base $2^{L}$ digits of $x$.

Finally, from Lemma 13 we have

$$
\operatorname{convl}(x, l, r, n)= \begin{cases}\operatorname{decr}\left(\operatorname{incr}\left(\operatorname{trim}(x, l, l, n), l, 2^{R(N+1)}-1, n\right),\right. & \\ \left.2^{R(N+1)}-1, r, n\right) & \text { if } R>L>0, \\ \operatorname{decr}(\operatorname{trim}(x, l, r, n), l, r, n) & \text { if } L>R>0, \\ \operatorname{trim}(x, l, r, n) & \text { if } L=R>0, \\ 0 & \text { otherwise. }\end{cases}
$$

and the functions trim and convl are normal by Lemmata 6-8 because incr and decr are normal. 
Lemma 16. For any set $F$ of polynomial growth functions and any normal class $C$, if $F^{\dagger^{c}} \subseteq C$ then $A C^{0}(F) \subseteq C$.

Proof. By lemmata 4, 11, 9, 12 and 15 we have conc, len, repl, ar $2 l$, convl $\in C$ and so $\operatorname{clos}_{S U B S T}\left(C_{1}\right.$, add, sub, and, conc, len, msp, ar $2 l$, repl, convl, $\left.F^{\dagger c}\right) \subseteq C$. The lemma follows immediately from the Quotient-free Basis Theorem of [6].

Corollary 17. For any set $F$ of polynomial growth functions,

$$
A C^{0}(F) \subseteq \operatorname{clos}_{S U B S T}\left(C_{1}, \text { add, sub, and, msp, rpt, } F^{\dagger c}\right) .
$$

Proof. By definition, $\operatorname{clos}_{S U B S T}\left(C_{1}, a d d, s u b, a n d, m s p, r p t, F^{\dagger c}\right)$ is a normal class which contains $F^{\dagger^{\dagger}}$.

Theorem 18 (Parametric Quotient-Free Basis Theorem). For any set F of polynomial growth functions, if $r p t \in A C^{0}(F)$ then

$$
A C^{0}(F)=\operatorname{clos}_{S U B S T}\left(C_{1}, a d d, s u b, \text { and, msp, rpt, } F^{\dagger c}\right) .
$$

Proof. Note that $C_{1}$, add, sub, and, msp $\in A C^{0}$, moreover $r p t \in A C^{0}(F)$ by hypothesis and $F^{\dagger_{c}} \subseteq A C^{0}(F)$ by Lemma 8 of [6].

\section{A new basis for $A C^{0}(F)$}

In this section we will prove the Improved Quotient-free Basis Theorem. In order to do so, we just need to show that $r p \in A C^{0}$ and to set $r p t=r p$ in Theorem 18.

Lemma 19. $r p(x, l, n) \in A C^{0}$.

Proof sketch. Recall that the predicate $A C^{0}{ }_{-} S U M(x, l, n)$ introduced in the Preliminaries is defined as

$$
A C^{0}{ }_{-} S U M(x, l, n) \Leftrightarrow(l n>0) \wedge\left(P_{1}(x, l, n) \vee P_{2}(x, l, n) \vee P_{3}(x, l, n)\right)
$$

are $P_{1}, P_{2}$ and $P_{3}$ are mutually disjoint $A C^{0}$ predicates. We show that $r p$ can be defined by cases. Indeed, there are functions $h_{1}, h_{2}$ and $h_{3}$ in $A C^{0}$ such that $r p(x, l, n)=h_{i}(x, l, n)$ if $P_{i}(x, l, n) \wedge l n>0$ is true. We assume that $l n>0$ and set $L=|l|$ and $N=|n|$.

First, assume that $P_{1}(x, l, n)$ is true. Then $x=\langle\overbrace{1, \ldots, 1}^{L-\text { times }} ; L\rangle \wedge(1<l)$ and

$$
\begin{aligned}
r p(x, l, n) & =\langle 1, \ldots, L \dot{-} 1, L, L \dot{-} 1, \ldots, 1 ; L\rangle \\
& =\langle 1, \ldots, L \dot{-} 1, L ; L\rangle \cdot 2^{L(L-1)}+\langle L \dot{-} 1, \ldots, 1 ; L\rangle \\
& =(\operatorname{repl}(L, l, l) \dot{-} \operatorname{arl}(l)) \cdot 2^{L(L-1)}+m s p(\operatorname{arl}(l), l) .
\end{aligned}
$$

Assume that $P_{2}(x, l, n)$ is true and recall that $2 t_{m}=m(m+1)$.

Then $x=\langle L-1, \ldots, 1,0 ; L\rangle \wedge(1<l)$ and

$$
\begin{aligned}
r p(x, l, n) & =\left\langle t_{L \dot{-1}-} \dot{-} t_{L-2}, \ldots, t_{L-1} \dot{-} t_{2}, t_{L \dot{-1}} \dot{-} t_{1}, t_{L-1}, \ldots, t_{1}, t_{0} ; L\right\rangle \\
& =\left\langle t_{L \dot{-1}} \dot{-} t_{L \dot{-} 2}, \ldots, t_{L-1} \dot{-} t_{2}, t_{L \dot{-1}} \dot{-} t_{1} ; L\right\rangle \cdot 2^{L(L-1)}+\left\langle t_{L \dot{-1}}, \ldots, t_{1}, t_{0} ; L\right\rangle \\
& =\left(\operatorname{repl}\left(t_{L-1}, l,\lfloor l / 2\rfloor\right) \dot{-} T(\lfloor l / 2\rfloor)\right) \cdot 2^{L(L-1)}+T(l)
\end{aligned}
$$


where $T(l)=\lfloor(\operatorname{ar} 2 l(l)+\operatorname{arl}(l)) / 2\rfloor=\left\langle t_{L-1}, \ldots, t_{0} ; L\right\rangle$ belongs to $A C^{0}$ by Lemma 10 and Lemma 19 of [6].

Assume that $P_{3}(x, l, n)$ is true and recall that this is equivalent to

$$
x<2^{|l||n|} \wedge \forall_{i<j<|n|}\left(x 2^{|l| i} \wedge x 2^{|l| j}=0\right) .
$$

Then, for any $k<2|l||n|$ there is at most one index $i<N$ such that $\operatorname{bit}\left(x 2^{|l| i}, k\right)=1$ and so, no carry is generated in the computation of $\sum_{i<|n|} x 2^{|l| i}$. Therefore,

$$
r p(x, l, n)=x \cdot \sum_{i<|n|} 2^{|l| i}=\sum_{i<|n|} x 2^{|l| i}=\bigvee_{i<|n|} x 2^{|l| i}
$$

where $\bigvee_{i<|n|} f(x, i)$ is defined as

$$
\operatorname{bit}\left(\bigvee_{i<|n|} f(x, i), j\right)=1 \Leftrightarrow \exists_{i<|n|} \operatorname{bit}(f(x, i), j)=1
$$

and belongs to $A C^{0}$ because $A C^{0}$ is closed with respect to sharply bounded quantifiers, see [2].

Concluding, $r p$ is defined by cases from $A C^{0}$ functions and predicates and so it belongs to $A C^{0}$.

We apply now Theorem 18 to obtain the Improved Quotient-free Basis Theorem.

Theorem 20. For any set $F$ of polynomial growth functions,

$$
A C^{0}(F)=\operatorname{clos}_{S U B S T}\left(C_{1}, a d d, s u b, \text { and, } m s p, r p, F^{\dagger c}\right) .
$$

Proof. Set $r p t=r p$ in Theorem 18. The theorem follows immediately from Lemma 19.

Corollary 21. $A C^{0}=\operatorname{clos}_{S U B S T}\left(C_{1}\right.$, add, sub, and, $\left.m s p, r p\right)$.

\section{New bases for $T C^{0}$}

In this section we show that both $\left\{C_{1}\right.$, add, sub, and, msp, $\left.\sum_{i<|n|} 2^{|l| i}, q u a d\right\}$ and $\left\{C_{1}, a d d, s u b\right.$, and, $\left.m s p, x \cdot \sum_{i<|n|} 2^{|l| i}\right\}$ are bases for $T C^{0}$. This result is independent from the striking result of [4], namely integer division is in $T C^{0}$, which was used in [7] to introduce the first basis for $T C^{0}$. The new bases are obtained as another application of Theorem 18.

\section{Lemma 22.}

$$
\left\{x y, x \cdot \sum_{i<|n|} 2^{|l| i}\right\} \cup A C^{0} \subseteq \operatorname{clos}_{S U B S T}\left(C_{1}, \text { add, sub, and, msp, } \sum_{i<|n|} 2^{|l| i}, q u a d\right) .
$$

Proof. Note first that $x y=(x+y)^{2}-x^{2}-y^{2}$. The lemma follows from Corollary 17 by setting $F=\emptyset$ and $\operatorname{rpt}(x, l, n)=x \cdot \sum_{i<|n|} 2^{|l| i}$. 
Now, we show that

$$
\operatorname{quad}^{\dagger c} \in \operatorname{clos}_{S U B S T}\left(C_{1}, \text { add, sub, and, msp, } \sum_{i<|n|} 2^{|l| i}, q u a d\right) .
$$

By Theorem 18, this implies that $\left\{C_{1}\right.$, add, sub, and, msp, $\left.\sum_{i<|n|} 2^{|l| i}, q u a d\right\}$ is a basis for $T C^{0}$.

Lemma 23 (Lemma 3.4 of [5]). $x^{2}=\sum_{i<|x|, b i t(x, i)=1}\left(2 \cdot 4^{i} \cdot \operatorname{MSP}(x, i)-4^{i}\right)$.

Proof. By induction on $x$, using the following definition of the quadratum function:

$$
\begin{aligned}
0^{2} & =0 \\
(2 y)^{2} & =4 y^{2} \\
(2 y+1)^{2} & =4 y^{2}+4 y+1 .
\end{aligned}
$$

Lemma 24. $q_{u a d}^{\dagger_{c}} \in \operatorname{clos}_{S U B S T}\left(C_{1}\right.$, add, sub, and, msp, $\left.\sum_{i<|n|} 2^{|l| i}, q u a d\right)$.

Proof. Set $R=|r|$ and $N=|n|$. Let $\mathbf{x}=x_{N}, \ldots, x_{1}$ and assume that $x_{j}^{2}<2^{R}$ for any $1 \leq j \leq N$. Consider the function

$$
f(x, y)= \begin{cases}2 \operatorname{MSP}(x, y) 4^{\min (|x|, y)}-4^{\min (|x|, y)} & \text { if } \operatorname{bit}(x, y)=1 \\ 0 & \text { otherwise }\end{cases}
$$

and note that $f$ is in $A C^{0}$ because $4^{\min (|x|, y)}=2^{\min (|x * x|, 2 y)} \in A C^{0}$. Then, $f^{\dagger^{\dagger}} \in A C^{0}$ by Lemma 8 of [6]. Furthermore, consider the functions

$$
M(r, n)=\operatorname{convl}\left(\operatorname{arl}(r), r, 2^{R N}-1, r\right) \sum_{i<N} 2^{R i}
$$

and

$$
g(x, r, n)=f^{\dagger_{c}}\left(\operatorname{repl}\left(x, 2^{R N}-1, r\right), M(r, n), r, 2^{R N}-1\right)
$$

belonging to $A C^{0}$ such that

$$
M(r, n)=\langle\overbrace{R-1, \ldots, R-1}^{N-\text { times }}, \ldots, \overbrace{0, \ldots, 0}^{N-\text { times }} ; R\rangle
$$

and

$$
g(\langle\mathbf{x} ; R\rangle, r, n)=\left\langle\left\langle u_{R-1, N}, \ldots, u_{R-1,1} ; R\right\rangle, \ldots,\left\langle u_{0, N}, \ldots, u_{0,1} ; R\right\rangle ; R N\right\rangle
$$

where $u_{i, j}=\left\{\begin{array}{ll}2 \operatorname{MSP}\left(x_{j}, i\right) 4^{i}-4^{i} & \text { if } \operatorname{bit}\left(x_{j}, i\right)=1 \\ 0 & \text { otherwise }\end{array}\right.$.

So, for $\left\langle s_{2 R-1}, \ldots, s_{1} ; R N\right\rangle=g(\langle\mathbf{x} ; R\rangle, r, n) \cdot \sum_{i<R} 2^{R N i}$ we have

$$
s_{R}=\left\langle x_{N}^{2}, \ldots, x_{1}^{2} ; R\right\rangle
$$


because

$$
s_{R}=\sum_{i<R}\left\langle u_{i, N}, \ldots, u_{i, 1} ; R\right\rangle=\left\langle\sum_{i<R} u_{i, N}, \ldots, \sum_{i<R} u_{i, 1} ; R\right\rangle
$$

by Lemma 10 and, for any $1 \leq j \leq N$,

$$
\sum_{i<R} u_{i, j}=\sum_{i<R, b i t\left(x_{j}, i\right)=1} 2 \operatorname{MSP}\left(x_{j}, i\right) 4^{i}-4^{i}=x_{j}^{2}
$$

by Lemma 23. Therefore, for

$$
q(x, r, n)=l s p\left(m s p\left(g(x, r, n) \cdot \sum_{i<R} 2^{R N i}, 2^{(R-1) R N}-1\right), 2^{R N}-1\right)
$$

we have $q(\langle\mathbf{x} ; R\rangle, r, n)=\left\langle x_{N}^{2}, \ldots, x_{1}^{2} ; R\right\rangle$. The lemma follows by noting that

$$
\begin{array}{r}
q u a d^{\dagger c}(x, l, n)=\operatorname{convl}\left(\operatorname{trim}\left(q\left(\operatorname{convl}\left(x, l, 2^{2 L}-1, n\right), 2^{2 L}-1, n\right), 2^{2 L}-1, l, n\right)\right. \\
\left., 2^{2 L}-1, l, n\right)
\end{array}
$$

where trim is the $A C^{0}$ function defined in Lemma 15 .

Now we obtain two new bases for $T C^{0}$.

Theorem 25. $T C^{0}=\cos _{S U B S T}\left(C_{1}\right.$, add, sub, and, msp, $\sum_{i<|n|} 2^{|l| i}$, quad $)$.

Proof. Set $F=\{q u a d\}$ and $\operatorname{rpt}(x, l, n)=x \cdot \sum_{i<|n|} 2^{|l| i}$. Then, by Theorem 18 and Lemma 24,

$$
\begin{aligned}
A C^{0}(\text { quad }) & =\operatorname{clos}_{S U B S T}\left(C_{1}, \text { add }, \text { sub, and, msp, } x \cdot \sum_{i<|n|} 2^{|l| i}, q u a d^{\dagger c}\right) \\
& \subseteq \cos _{S U B S T}\left(C_{1}, \text { add }, \text { sub }, \text { and }, \text { msp }, \sum_{i<|n|} 2^{|l| i}, q u a d\right) \\
& \subseteq T C^{0}
\end{aligned}
$$

and the theorem follows immediately because $A C^{0}($ quad $)=A C^{0}($ mult $)=T C^{0}$.

Theorem 26. $T C^{0}=\operatorname{clos}_{S U B S T}\left(C_{1}, a d d\right.$, sub, and, msp, $\left.\left.x \cdot \sum_{i<|n|}\right|^{|l| i}\right)$.

Proof. By Theorem 25 it suffices to show that

$$
\text { quad } \in \cos _{S U B S T}\left(C_{1}, \text { add, sub, and, msp, } x \cdot \sum_{i<|n|} 2^{|l| i}\right) .
$$

First, consider the function $f_{1}(x, y)=\operatorname{convl}\left(y, 2,2^{|x|+|y|+1}, y\right)$ such that

$$
f_{1}(x, y)=\left\langle y_{|y|-1}, \ldots, y_{0} ;|x|+|y|+1\right\rangle
$$

where $y_{i}=\operatorname{bit}(y, i)$ and the function $f_{2}(x, y)=\operatorname{repl}\left(x, 2^{|x|+|y|+1}, y\right)$ such that

$$
f_{2}(x, y)=\langle\overbrace{x, \ldots, x}^{|y|-\text { times }} ;|x|+|y|+1\rangle \text {. }
$$


Then, for $f_{3}(x, y)=\left(2^{|x|}-1\right) \cdot f_{1}(x, y) \wedge f_{2}(x, y)$ we have by Lemma 20 of [6]

$$
\begin{aligned}
f_{3}(x, y) & =\left\langle x y_{|y|-1}, \ldots, x y_{0} ;|x|+|y|+1\right\rangle \\
& =\left\langle x y_{|y|-1} 2^{|y|-1}, \ldots, x y_{0} ;|x|+|y|\right\rangle .
\end{aligned}
$$

Note that $f_{1}, f_{2}$ and $f_{3}$ belong to $A C^{0}$ and therefore to $\operatorname{clos}_{S U B S T}\left(C_{1}, a d d, s u b\right.$, and, $\left.m s p, x \cdot \sum_{i<|n|} 2^{|l| i}\right)$ by Corollary 17.

Finally, for $f_{4}(x, y)=f_{3}(x, y) \cdot \sum_{i<|y|} 2^{\left|2^{|x||y|}-1\right| i}$ we have

$$
f_{4}(x, y)=\left\langle x y_{|y|-1} 2^{|y|-1}, \ldots, x y_{0} ;|x|+|y|\right\rangle \cdot \sum_{i<|y|} 2^{(|x|+|y|) i}
$$

and the theorem follows by Lemma 10 because the $|y|$-th digit in base $2^{|x|+|y|}$ of $f_{4}(x, y)$ is $\sum_{i<|y|} x y_{i} 2^{i}=x y$.

Remark. The difference between $A C^{0}$ and $T C^{0}$ seems to be very subtle. Indeed, the basis for $A C^{0}$ and the basis for $T C^{0}$ of Theorem 26 differ for one function only. Moreover, the former basis contains $r p$ while the latter basis contains $x \cdot \sum_{i<|n|} 2^{|l| i}$, which is a sort of "extension" of $r p$. This result could be the starting point for a new, algebraic proof that $A C^{0} \neq T C^{0}$.

\section{Bases for complexity classes with complete problems}

The new bases introduced in Section 4 and Section 5 can be used to obtain bases for complexity classes with complete problems. Indeed, in [6] it was shown that a function class $F$ with complete decision problems under $A C^{0}$ reductions can be characterized as the $A C^{0}$ closure of the characteristic function of a suitable complete problem, provided that $F$ is closed with respect to substitution and CRN. Then, the Improved Quotient-free Basis Theorem yields immediately a new basis for $F$. Here we state the new bases without proofs. The interested reader may refer to Section 3 of [6] for a full treatment of the subject.

\section{Theorem 27.}

$$
\begin{gathered}
N C^{1}=A C^{0}\left(\operatorname{ch}_{B F V P}\right)=\operatorname{clos}_{S U B S T}\left(C_{1}, a d d, s u b, \text { and, msp }, r p, c h_{B F V P}^{\dagger_{c}}\right), \\
L=A C^{0}\left(\operatorname{ch}_{1 G A P}\right)=\operatorname{clos}_{S U B S T}\left(C_{1}, a d d, s u b, \text { and }, m s p, r p, c h_{1 G A P}^{\dagger_{c}}\right), \\
P=A C^{0}\left(\operatorname{ch}_{C V P}\right)=\operatorname{clos}_{S U B S T}\left(C_{1}, a d d, s u b, \text { and }, m s p, r p, c h_{C V P}^{\dagger_{c}}\right), \\
P S P A C E=A C^{0}\left(c_{Q B F}\right)=\operatorname{clos}_{S U B S T}\left(C_{1}, a d d, s u b, \text { and }, m s p, r p, c h_{Q B F}^{\dagger_{c}}\right)
\end{gathered}
$$

where BFVP is the Boolean Formula Value Problem, 1 GAP is the Degree-One Graph Accessibility Problem, CVP is the Circuit Value Problem and QBF is the Quantified Boolean Formulas Problem.

Note that Theorem 27 also holds when $r p$ is replaced by $\sum_{i<|n|} 2^{|l| i}$ and quad or by $x \cdot \sum_{i<|n|} 2^{|l| i}$ (and $B F V P, 1 G A P, C V P$ and $Q B F$ are possibly replaced by $T C^{0}$-complete problems for $N C^{1}, L, P$ and $P S P A C E$ respectively). 


\section{References}

[1] P. Clote. Sequential, machine-independent characterizations of the parallel complexity classes AlogTIME, $A C^{k}, N C^{k}$ and $N C$, pages 49-69. Birkhäuser Boston, Boston, MA, 1990.

[2] P. Clote. Computation models and function algebras. In Edward R. Griffor, editor, Handbook of Computability Theory, volume 140 of Studies in Logic and the Foundations of Mathematics, pages 589 - 681. Elsevier, 1999.

[3] P. Clote and G. Takeuti. First Order Bounded Arithmetic and Small Boolean Circuit Complexity Classes, pages 154-218. Birkhäuser Boston, Boston, MA, 1995.

[4] W. Hesse, E. Allender, and D. A. Mix Barrington. Uniform constant-depth threshold circuits for division and iterated multiplication. Journal of Computer and System Sciences, 65(4):695 - 716, 2002. Special Issue on Complexity 2001.

[5] S. Mazzanti. CRN elimination and substitution bases for complexity classes. Fundam. Inform., 120(1):29-58, 2012.

[6] S. Mazzanti. Bases for $\mathrm{AC}^{0}$ and other complexity classes. Fundam. Inform., 136(4):433-460, 2015.

[7] S. A. Volkov. Generating some classes of recursive functions by superpositions of simple arithmetic functions. Doklady Mathematics, 76(1):566-567, 2007.

[8] S. A. Volkov. Finite bases with respect to the superposition in classes of elementary recursive functions, dissertation. CoRR, abs/1611.04843, 2016. 